ORIGINAL ARTICLE

\title{
Implications of carrier identification in newborn screening for cystic fibrosis
}

\author{
E P Parsons, A J Clarke, D M Bradley
}

Arch Dis Child Fetal Neonatal Ed 2003;88:F467-F471

See end of article for authors' affiliations

.....................

Correspondence to: Dr Parsons, SONMS UWCM, Heath Park, Cardiff, Wales CF14 4XN, UK; parsonsep@ cardiff.ac.uk

Accepted

13 December 2002
Objective: To investigate the psychosocial implications for families whose infant was identified as a cystic fibrosis carrier by newborn screening.

Design: Prospective psychosocial assessment.

Setting: Primary care.

Respondents: Study: (a) families of an affected infant identified by screening $(\mathrm{n}=9)$; (b) families of a carrier infant identified by screening $(n=10)$. Control: group of mothers from the general population ( $\mathrm{n}=82$ ).

Interventions: Questionnaires and semistructured interviews.

Main outcome measures: Attitude to screening, assessments of the mother/baby relationship, anxiety, wellbeing.

Results: All families were in favour of screening, with no evidence that the mother/baby relationship, anxiety or wellbeing had been adversely affected. Parents, however, did identify problems in terms of the service delivery protocol and genetic counselling practice.

Conclusion: Six months after disclosure, carrier identification was not perceived by parents to be problematic.
$\mathrm{N}$ ewborn screening for cystic fibrosis (CF) has been possible since 1979 but remains controversial because, although many paediatricians see value in earlier prophylactic interventions, these have not yet been shown, under trial conditions, to provide substantial long term benefits. ${ }^{1}$

Currently in the United Kingdom, about 20\% of newborns are screened for CF (Northern Ireland, Wales, and parts of England), but in April 2001 Yvette Cooper, Minister for Public Health, announced that, subject to advice from the National Screening Committee, screening would be extended to all areas of England. ${ }^{2}$ Screening has also been proposed for Scotland. ${ }^{3}$

All screening protocols assay immunoreactive trypsinogen in dried blood spots as the primary test. Approximately $0.5 \%$ of babies will have immunoreactive trypsinogen levels above a threshold value, but only 1 in 12 of these will be affected. To reduce this high false positive rate, most laboratories have refined the screening process by introducing mutation analysis on the original blood sample. This inevitably identifies some healthy carrier infants. The population with an abnormal immunoreactive trypsinogen level is enriched with carriers and contains twice the number that could be expected (unpublished Welsh data). The number of carriers identified will depend on the range of mutations included in the analysis, but will be about one case for each affected infant detected.

The identification of healthy carrier infants is seen by some health professionals as undesirable because of its potential for early stigmatisation ${ }^{4}$ and disruption to the mother/baby relationship. ${ }^{5}$ Some policy makers, both in the United Kingdom and abroad, regard such identification as a major problem, and this is impeding the wider introduction of CF screening (R J Pollitt, personal communication, 2002).

This paper reports the findings of a small pilot study carried out in 1999 to investigate the psychosocial implications for families with an abnormal screening result, in particular, families whose infant was identified as a carrier.

\section{METHODS}

During the research period, screening samples were received from 32090 babies; 32034 were screened for CF; 56 families declined the test. When screening for CF was introduced in Wales, an initial protocol for service delivery was developed drawing on our experience in screening for Duchenne muscular dystrophy. ${ }^{6}$ This protocol for the follow up of abnormal results is detailed in the box. There were 19 abnormal results, after sweat testing (nine were identified as having $C F$, and 10 as carriers). All the families agreed to join the psychosocial study. In addition, a group of mothers from the general population $(\mathrm{n}=82)$ was drawn to provide control data where appropriate.

To investigate the psychosocial implications of newborn screening for $\mathrm{CF}$, three research interventions were used. Firstly, an assessment by the health visitor of the mother's responses to her baby on five dimensions: feeding, interest, response, speech, and touch. ${ }^{7}$ The assessment was made before the query about CF was raised and one month later. Each dimension was scored from 0 to 2 , giving a cumulative score ranging from 0 to 10 . Secondly, a structured questionnaire was completed by mothers six months after sweat testing. The following measures were included:

(a) Attitude to screening (options given were in favour, against, undecided).

(b) Rejection index ${ }^{8}$ : mothers were asked to score the statement that explored potential rejection ("I feel I want to run away and leave my baby"). The statement was scored l (strongly disagree) to 5 (strongly agree) giving a range of $1-5$.

(c) Protection index ${ }^{8}$ : mothers were asked to score four statements that explored potential overprotection (feeling protective, wanting to hug, watching closely, and not trusting baby with others). Each statement was scored 1 (strongly disagree) to 5 (strongly agree) giving a range of $4-20$. 
(d) Baby adjectives ${ }^{8}$ : mothers were given a list of 14 adjectives and asked to select as many as they thought best described their baby. The adjectives were: doing well, healthy, placid, responsive, alert, little, demanding, difficult, miserable, great, lovely, fun, cuddly, and exhausting.

(e) Anxiety: mothers completed the six item short form of the state scale ${ }^{9}{ }^{10}$ which has a range of 6-24 with a normal range of $6-12$.

(f) Wellbeing: mothers completed the general health questionnaire (GHQ 12). ${ }^{11}$ The GHQ was scored conventionally and has a range of $0-24$ with a normal range of $0-4$.

Data from the control group were available for items b, c, d, and $\mathrm{f}$.

Thirdly, a semistructured interview with each family six months after sweat testing. The aim of the informal interview was to explore the advantages and disadvantages of newborn screening and parent's experiences of the process.

The quantitative data were analysed using SPSS for Windows version 10. Statistical analysis was carried out using Kruskal-Wallis analysis of variance, $t$ test, MannWhitney, Wilcoxon, $\chi^{2}$, and Fisher's tests as appropriate. The qualitative data were transcribed and then analysed to identify critical themes. The two data sets were then compared and combined. The results are reported with particular reference to the implication for carrier families, using the following headings: attitude to newborn screening, infant carrier identification, adult carrier identification, newborn screening protocol, and the family dimension.

\section{RESULTS}

\section{Attitude to screening}

All the families who had their baby identified as a carrier $(\mathrm{n}=10)$ were in favour of newborn screening. Typical comments were:

\section{"...glad I know...I would rather know than not know"} (051)

"...sooner things come to light the better...at least we know it's in the family now." (034)

"In favour definitely, we can be aware of that for when they start their family." (042)

\section{Carrier infant identification}

Health visitor assessment of the mother/baby relationship (FIRST scores)

There was no evidence of any significant change (Wilcoxon) in the mother/baby relationship using the FIRST assessment scores.

Rejection index, protection index, anxiety, and wellbeing

No significant differences ( $t$ test, Mann-Whitney) were found between the CF carrier cohort, the CF affected cohort, and the control group (where appropriate) on the rejection index, the protection index, anxiety scores, or wellbeing. It is interesting to note, however, that, on the protection index, the affected cohort scored higher than the other cohorts on the statements about trusting their baby with others and watching closely. This is perhaps a reflection of the fact that their babies were symptomatic.

Table 1 gives the results of these measures.
Baby adjectives

There was no significant difference $\left(\chi^{2}\right.$, Fisher's) between the CF carrier cohort, CF affected cohort, and the control group in their choice of baby adjectives, although fewer mothers in the affected group chose the descriptor "healthy".

\section{Qualitative data}

The interview provided qualitative data on how the parents talked about their baby now they were known to be a carrier. During the thematic analysis, we searched for statements that might indicate concerns that their baby's identity had been spoiled or that they anticipated future stigmatisation.

The parents' accounts focused on three main issues. Firstly, they all made reference to the fact that their baby was "perfectly healthy":

\section{"I knew with him being just a carrier he wouldn't be ill"} (042)

"It didn't really bother me at all, knowing that they were healthy (047)

\section{"No I'm quite satisfied she's not going to suffer from it"} (046)

One family had taken their knowledge one step further:

\section{"He was well clear, he was a carrier but the genes were recessive" (036)}

In two families the adult carrier had made the connection between their own continued health and the fact their baby was going to be the same:

"I've lived a normal life up until now and it's not affected me so it shouldn't affect him" (047)

Secondly, the way the families talked about their baby's carrier status indicated that it caused them very little concern. They talked about them as being "just a carrier" or "only a carrier":

"He hasn't got it so it's put to the back of my mind" (036)

"As far as we're concerned it's finished-I know he's a carrier..." (051)

"I wasn't that concerned..." (046)

"...the pressure was off...I know that he is perfectly fine..." (042)

"I don't give it a second thought..." (044)

Thirdly, they were all aware that at some point in the future they would have to pass the information on:

"The thing is you're aware of it but for them you are talking 15 or 20 years ahead and there will be better forms of identification then" (034)

"... she was only a carrier but we will need to let her know at a certain age so she is more aware than I was" (041), 
Table 1 Median and interquartile range for FIRST scores, rejection index, protection index, anxiety, and wellbeing

\begin{tabular}{|c|c|c|c|}
\hline Measure & $\begin{array}{l}\text { Carrier cohort } \\
(n=10)\end{array}$ & $\begin{array}{l}\text { Affected cohort } \\
(\mathrm{n}=9)\end{array}$ & $\begin{array}{l}\text { Control } \\
(n=82)\end{array}$ \\
\hline \multicolumn{4}{|l|}{$\begin{array}{l}\text { FIRST scores } \\
\text { (normal range } 8-10 \text { ) }\end{array}$} \\
\hline Before & $10(8.3-10)$ & $10(8.5-10)$ & \\
\hline After & $9.5(8.3-10)$ & $10(10-10)$ & \\
\hline \multicolumn{4}{|l|}{ Rejection index } \\
\hline $\begin{array}{l}\text { (range 1-5) } \\
\text { Protection index }\end{array}$ & $1(1-1)$ & $1(1-1)$ & $1(1-2)$ \\
\hline (range 4-20) & $10.5(6-12.3)$ & $14(13-17.5)$ & $13.5(12-16)$ \\
\hline $\begin{array}{l}\text { Anxiety (normal } \\
\text { range 6-12) } \\
\text { Wellbeing (GHQ) }\end{array}$ & $11(9.5-12.3)$ & $11(10-14.5)$ & \\
\hline (normal range $0-4$ ) & $1(0-3.5)$ & $3(0-8)$ & $2.5(1-5)$ \\
\hline
\end{tabular}

There was also a feeling that at some point the young person would have to take their own decisions:

"I mean they may fall in love with someone who is also a carrier... but it's up to them at that point...that's their choice, not mine...that's their life...it's up to them and my other children to decide what they want to do." (036)

In summary, there was no evidence that the mother/baby relationship had been affected by the carrier identification or that carrier status was seen by parents as a problem in terms of spoiled identity.

\section{Adult carrier identification}

The inevitable consequence of a baby being identified as a CF carrier is that at least one parent is also a carrier. In this study, six of the fathers were carriers and four of the mothers. One mother recalled that when she first heard she had been a "bit apprehensive" (034), another said that initially she had been "gutted" (046). By the six month interview, however, all the carriers were very philosophical about their status:

"Of course it means something but it's not particularly good or bad" (041)

"It doesn't affect me, it's not an illness, l just carry on doing my normal thing" (042)

"It's just the same as blue eyes, tall, short whatever, I didn't feel anything was bad" (044)

"It's just one of those things I have, its latent, its just lying there..." (036)

For some carriers there was recognition they were not alone:

\footnotetext{
"There are so many people who are carriers, it's going to come up time and time again." (043)
}

"I am sure there are literally hundreds of people who are carriers, we're just not aware of it." (047)

There were two parents, however, who felt some guilt about what they might have passed on. One father, although he was not at all concerned about his own carrier state, said:
"I felt uncomfortable, I'd passed something on to my son" (044)

It was the same for one mother:

"You don't like to think you are going to pass anything awful to a child, I don't feel bad about it now but I did have this guilty feeling..." (034)

For other carriers there was no problem, one mother said:

"...no I've got no guilt...I don't blame my mother or father for passing it on to me... it's blameless isn't it. They say it's the most common hereditary...goodness knows what else we've got laying dormant... (036)

\section{Newborn screening protocol}

When parents were asked about the emotions they had felt at that time, they talked about "feeling afraid" and "being worried"; one mother described it "like a dark cloud" (036), another talked about being "in a state" (043), and another referred to it as a "shock" (041). One distressing aspect for some of the families with a carrier infant was the way they had been told that there was a query on the CF test.

Further analysis of the transcripts identified three specific areas where anxieties had been unnecessarily raised because of the way the case had been handled. Firstly, on four occasions two health professionals had called on the family to raise the query: the health visitor and a specialist CF nurse. For these families, this visit by a specialist to talk about CF just confirmed their worst fears:

"I was under the impression that he really had it. Having a counsellor come all the way from (name of town) to go through it all before having the sweat test, I was convinced that he had it" (042).

One mother had been told the information over the telephone by her general practitioner, and another mother first learnt about the query when she received a card giving her an appointment with a paediatrician five days later, her health visitor having not been informed. Although a protocol was in operation during the research period, for various reasons, it was not always followed.

Secondly, whether the families had been visited by their health visitor or the specialist nurse, they had been given details about CF. In some cases they had been left with a CF Trust leaflet:

\section{"So I read the leaflet and automatically thought the worst It was saying what CF was..." (047)}

"She just explained a bit about what the disease was-so we got the basics... all I could see was the fact that there was something seriously wrong with him" (051).

Thirdly, most families recalled the negative language that had been used:

\section{"It's just the shock of someone telling you there is something wrong" (041)}

"She just said that the test had come back positive" (042) 
For one mother it had been the approach that had been hard to cope with:

"I was not happy with the way it was put across... they just showed no sympathy, no warning, they just blurted it out" (046).

\section{The family dimension}

The identification of any genetic disease has family implications. In this study all of the carrier families were offered and accepted genetic counselling. There were, however, two particular areas that they highlighted as being problematic: the testing of their other children and the difficulty of telling family members.

\section{Testing other children}

The study has shown that carrier status, per se, caused little concern to the families, either in terms of the infant or adult carrier. It was therefore difficult for parents to understand why clinical geneticists were reluctant to test their other children. One mother said:

"They said if he wanted it done when he was older, he could, and his partner as well, but they wouldn't do it now.... They said you're better off doing it when he's older but I would rather know now than later on" (043)

It was the same for another mother:

\section{"Well the letter said that I could have them tested when they were older but I would rather know now when they're younger" (046)}

One mother, who had been worried about her daughter, was told she could only have her tested if she and her husband were both carriers.

\section{The extended family}

Most families talked at length about passing the news to other family members. The language, however, reflected feelings of "obligation", with the use of phrases such as: "it meant contacting", "I had to pass information on", "we felt we should", "it's our responsibility to tell". In some cases making these contacts had been the most difficult part of the whole event. Brothers, step-brothers, sisters, and step-sisters, some of whom were pregnant when the carrier status had been identified, needed to be told. For one grandmother, the concern was not her own potential carrier status, but the fact that she might have to contact her ex-partner, the father of the index carrier:

\section{"She's not very worried herself but she doesn't want to contact him unless she has to...that's the only thing she's worried about." (046)}

In another family, it had led to difficulties between the grandparents. For another, the brother, having been told, would not talk about it, and his carrier sister felt a distance had developed between them not previously there.

One mother said:

\footnotetext{
"We thought we were under an obligation to be tested for members of the family more than anything else... it was still a family sort of thing... and I just feel that we got no
}

support... it was just very difficult... the emotional aspect of it..." (034)

Another couple felt that they did not have enough information and were struggling to find the right words:

"I knew I had to tell my Dad and he rang my brothers who
live away... we all met up and we tried to talk to them but
it was difficult getting it through... I am sure we put both
feet in it somewhere... missed out relevant points..." (044)

It was interesting that, for many families, discussion about carrier issues in the wider family proved to be most problematic consequence of newborn screening.

\section{DISCUSSION}

This small study has indicated that all the families who had their baby identified as a carrier were in favour of newborn screening, and there was no evidence that the mother/baby relationship, maternal anxiety, or wellbeing had been adversely affected. These findings were supported by the qualitative data, with no evidence that they were either concerned about the health of their baby or possible future stigmatisation. This finding is different from that of Baroni et al. ${ }^{5}$ In their study of families, one year after a carrier result, they report that, although parents had low stress scores, they showed a degree of defensiveness which could reflect "hypervigilance and emotional repression".

Over the past 15 years, there has been extensive research looking at the psychosocial implications of carrier screening for adults. Most studies have concluded that, when a carrier is identified, there are raised anxieties, but within one year these have dissipated. ${ }^{12-14}$ Adults in this study were different, they had not chosen to have carrier screening and were therefore not expecting a result that would implicate them. Our findings are in line with other studies, in that after six months there was no evidence that any of the parents were unduly concerned.

This study, however, has highlighted new findings in terms of problems in two areas of service delivery: the protocol used by health professionals for raising the query with families and the delivery of genetic counselling. Firstly, some families reported that they had experienced unnecessary anxiety because of the way they had been told there was a query on the newborn screening test. This study has been valuable in identifying those areas where the protocol for CF needs some modification and more rigorous implementation. In the case of those families where a single mutation is found we would recommend that:

(1) the family are visited to be told about the query by a member of their primary healthcare team who is known to them and is not accompanied by a specialist CF nurse who they have not met before;

(2) the health professional concerned takes great care to use positive language and be reassuring;

(3) at the initial meeting any details about CF as a disease are kept to a minimum.

Secondly, with respect to the delivery of genetic counselling services, parental accounts highlighted their concerns about being unable to ascertain the carrier status of their other children and the problems they faced in talking about carrier issues to other members of their family. The Working Party of the Clinical Genetics Society $(\mathrm{UK})^{4}$ concluded that carrier testing of children should be deferred until they are of an age to be fully involved in the decision. The feedback from the families would indicate that this is current practice in 
Protocol for the follow up of an abnormal newborn screening test for CF

1. Contact primary healthcare team (health visitor and general practitioner) to inform them, to discuss health of baby, and arrange for them to talk to family about having baby sweat tested.

2. Inform the secondary healthcare team (paediatrician and specialist CF nurse), arrange date for sweat test, and suggest liaison between specialist nurse and health visitor. 3. Family told about the query and the arrangements for the sweat test.

4. Sweat testing and result given by paediatrician on the same day, implications discussed.

5. Referral to medical genetics for counselling offered.

departments of medical genetics. In terms of carrier testing therefore parents are receiving a double message. From genetics, they are told it is not policy to disclose children's carrier status to parents whereas, from newborn screening, it is apparently quite acceptable and our research indicates that the information has not caused them any distress. Clearly this is an area of policy that needs clarification.

The second area some parents found difficult to handle was the expectation they would initiate discussions with their extended family. It is interesting that the carrier identification of an infant and its parent seemed to raise minimal concerns whereas, for some, talking about it to other family members was problematic. Fanos and Johnson, ${ }^{15}$ in their study of CF siblings, and Williams and Schutte ${ }^{16}$ and Claes et al $^{17}$ report similar findings. It would seem as if the discomfort is caused because a genetic diagnosis has the potential to bring to the surface dysfunctional family relationships and create new tensions. This problem, however, is not specific to newborn screening for CF, and we would recommend that a protocol is developed to cover all situations where cascade testing takes place. The protocol should ensure that members of an index family are not left unsupported as the main information givers and are provided with a short information leaflet which they can pass on to their relatives. This information sheet should contain details about the condition, the purpose of cascade screening, together with contact names and telephone numbers. Such a factsheet is already available from the Cystic Fibrosis Trust. ${ }^{18}$

\section{Conclusion}

This small study has provided important preliminary data on the psychosocial implications of carrier identification as a result of newborn screening. The data indicate that, six months after disclosure, carrier identification was not perceived by parents to be problematic. There was, however, evidence of areas where healthcare policy needs clarification and health professionals' practice needs modification.

\section{ACKNOWLEDGEMENTS}

We would like to thank all the families who participated in the research, Dr Kerry Hood for statistical support, and Carolyn Eason for data collection.

This research was funded by Wales Office of Research and Development.

\section{Authors' affiliations}

E P Parsons, A J Clarke, Institute of Medical Genetics, University of Wales College of Medicine, Heath Park, Cardiff CF14 4XN, Wales, UK D M Bradley, Department of Medical Biochemistry, University Hospital of Wales, Heath Park, Cardiff CF14 4XW, Wales, UK

\section{REFERENCES}

1 Merelle ME, Nagelkerke AF, Lees CM, et al. Newborn screening for cystic fibrosis (Cochrane Review). Cochrane Library. Issue 2. Oxford: Update Software, 2002.

2 Department of Health. Boost for baby health: new screening programmes for cystic fibrosis and hearing impairments in newborns. Press release 2001/ 0208. http://info.doh.gov.uk/doh/intpress.nst/page/2001-0208.

3 Scottish Executive Health Department. NHS HDL (2001) 73: neonatal screening for cystic fibrosis. Scottish Executive Health Department, 2001

4 Clinical Genetics Society (UK). The genetic testing of children. Report of Working Party of the Clinical Genetics Society (UK). J Med Genet 1994;72:785-97.

5 Baroni MA, Anderson YE, Mischler E. Cystic fibrosis newborn screening: impact of early screening results on parenting stress. Pediatr Nurs 1997;23:143-51

6 Parsons EP, Bradley DM, Clarke AJ. Disclosure of Duchenne muscular dystrophy after newborn screening. Arch Dis Child 1996;74:550-3.

7 Salariya EM, Cater Jl. Mother-child relationship-FIRST score. J Adv Nurs 1984;9:589-95.

8 Parsons EP, Clarke AJ, Hood K, et al. Newborn screening for Duchenne muscular dystrophy: a psychosocial study. Arch Dis Child 2002;86:F91-5.

9 Speilberger CD. Manual for the State-Trait Personality Inventory STAI (Form Y). Palo Alto, CA: Consulting Psychologists Press, 1983.

10 Marteau TM, Bekker $\mathrm{H}$. The development of a six-item short-form of the state scale of the Spielberger state-trait anxiety inventory (STAI). Br J Clin Psychol 1992;31:301-6.

11 Goldberg DP. The detection of psychiatric illness by questionnaire. London: Oxford University Press, 1972.

12 Mennie M, Gilfillan A, Compton M, et al. Prenatal screening for cystic fibrosis. Lancet 1992;340:214-16.

13 Watson EK, Mayall ES, Lamb J, et al. Psychological consequences of community carrier screening programme for cystic fibrosis. Lancet 1992;340:217-20.

14 Bekker H, Denniss G, Modell M, et al. The impact of population screening for carriers of cystic fibrosis. J Med Genet 1994;31:364-8.

15 Fanos JH, Johnson JP. Barriers to carrier testing adult cystic fibrosis sibs: the importance of not knowing. Am J Med Genet 1995;59:85-91.

16 Williams JK, Schutte DL. Benefits and burdens of genetic carrier identification. West J Nurs Res 1997; 19:71-81.

17 Claes E, Evers-Kiebooms G, Boogaerts A, et al. Communication with close and distant relatives in the context of genetic testing for hereditary breast and ovarian cancer patients. Am J Med Genet 2003; in press.

18 Cystic Fibrosis Trust. The family cascade screening programme for cystic fibrosis: An information factsheet for relatives (and their partners) of people with Cystic Fibrosis. http://www.cftrust.org.uk, 2001. 